\title{
Is parathyroidectomy beneficial in patients with mild, asymptomatic primary hyperparathyroidism?
}

\author{
Claudio Marcocci and Aldo Pinchera
}

Most patients with primary hyperparathyroidism (PHPT) are asymptomatic and are incidentally recognized because a mild elevation of serum calcium and parathyroid hormone levels is observed (Silverberg SJ and Bilezikian JP [2001] Clinical presentation of primary hyperparathyroidism in the United States. In The Parathyroids: Basic and Clinical Concepts, 349-360 [Eds Bilezikian JP et al.] San Diego: Academic Press). It is unclear whether parathyroidectomy would be beneficial to these patients. Indeed, disease does not worsen in most of them, although progression has been reported in $25 \%$ over a 10 -year period. In 1990 and 2002, a panel of experts wrote guidelines to advise clinicians which patients should be advised to have surgery and which should be followed up conservatively (Bilezikian JP et al. [2002] J Clin Endocrinol Metab 87: 5353-5361).

Three randomized clinical trials (Rao DS et al. [2004] J Clin Endocrinol Metab 80: 5415-5422; Bollerslev J et al. [2007] J Clin Endocrinol Metab 92: 1613-1615; Ambrogini E et al. [2007] J Clin Endocrinol Metab 92: 3114-3121) evaluated whether parathyroidectomy in asymptomatic patients who do not meet surgical guidelines shows beneficial effects compared with no surgery. BMD, quality of life (QOL; assessed by the Short Form 36 [SF-36] general health survey) and psychological function (assessed by the symptom checklist SCL90R) were evaluated.

All three studies showed increased femur BMD after surgery compared with the surveillance group, but the increase was statistically significant only in the studies of Rao et al. and Ambrogini et al.; two studies (Bollerslev et al. and Ambrogini et al.) reported a significant benefit at the lumbar spine. The increase in BMD occurred during the first 6 months after the operation at sites enriched in cancellous bone and could be due to rapid reduction of bone resorption and filling in of the enlarged remodeling space. Although the increase in BMD observed in these patients is comparable to that observed

\section{All three \\ studies showed \\ increased \\ femur BMD \\ after surgery...}

C Marcocci is an Associate Professor of the Department of Endocrinology and Metabolism at the University Hospital of Pisa, Pisa, Italy.

A Pinchera is an Advisory Board member of Nature Clinical Practice Endocrinology \& Metabolism.

\section{Competing interests}

The authors declared no competing interests.

www.nature.com/clinicalpractice doi:10.1038/ncpendmet0666 in osteoporotic postmenopausal women given oral bisphosphonates, it is unclear whether a reduction of fracture risk is likely to occur after parathyroidectomy in asymptomatic patients with PHPT.

Two of the studies (Rao et al. and Ambrogini et al.) found no difference in baseline QOL between patients with PHPT and healthy, control, nonhyperparathyroid individuals, but both studies showed a modest but significant beneficial effect on QOL as a result of parathyroidectomy. On the other hand, the study by Bollerslev et al. reported a lower QOL and more psychological symptoms at baseline in patients with PHPT than controls, and reported no significant benefit of surgery, even though an improvement was observed after parathyroidectomy in some QOL domains. The clinical relevance of these changes is unclear and a potential placebo effect of surgery might occur. The psychological tools used for assessment in these studies were not, moreover, specifically designed for patients with PHPT.

In patients with PHPT followed-up who did not undergo surgery, biochemical parameters and spine BMD did not significantly change; however, a mild, but statistically significant, decrease in hip BMD was observed by Rao et al. and Ambrogini et al. A significant worsening in some of the SF-36 domains was reported by Rao et al. and Bollerslev et al., but not by Ambrogini et al. According to Ambrogini et al., $23 \%$ of patients who did not undergo surgery developed at least one $\mathrm{NIH}$ criterion for surgery, an unexpected finding considering the short (1 year) follow up.

In patients with mild, asymptomatic PHPT, successful parathyroidectomy is followed by an improvement in BMD and in some parameters that reflect QOL. To what extent these beneficial changes after parathyroidectomy are worth the risk of undertaking surgery in such apparently healthy individuals remains to be established. 